J. Clin. Chem. Clin. Biochem.

Vol. 15,1977 , pp. $545-548$

\title{
Bindung von Cortisol, Fluocortolon und Difluocortolon an Humanplasmaproteine
}

\author{
Von H. Fellier, H. Gleispach und H. Esterbauer \\ Aus dem biochemischen Institut (Vorstand: Univ. Prof. Dr. E. Schauenstein) der Universität Graz und der \\ Universitäts-Kinderklinik (Vorstand: Univ. Prof. Dr. E. Zweymüller) Graz
}

(Eingegangen am 29. Juli 1976/3. März 1977)

\begin{abstract}
Zusammenfassung: Die Bindungseigenschaften von Humanplasma, Humanalbumin, Human- $\beta$ - und $\gamma$-Globulinen gegenüber $\left[{ }^{3} \mathrm{H}\right]$ Cortisol, $\left[{ }^{3} \mathrm{H}\right]$ Fluocortolon und $\left[{ }^{3} \mathrm{H}\right]$ Difluocortolon wurden mit Hilfe einer Gleichgewichtsdialyse untersucht. Cortisol in physiologischer Konzentration $(0,4 \mu \mathrm{mol} / \mathrm{l})$ liegt bei $25^{\circ} \mathrm{C}$ in Humanplasma zu $98 \%$, Fluocortolon zu $96 \%$, und Difluocortolon zu $85 \%$ an Protein gebunden vor. Cortisol wird unter diesen Bedingungen hauptsächlich vom corticosteroidbindenden Globulin (CBG, Transcortin) gebunden, Fluocortolon ist zu $2 / 3$ an CBG und $1 / 3$ an Albumin und Globuline gebunden. Difluocortolon wird hauptsächlich von Albumin und Globulinen, aber nicht vom CBG gebunden. Die Bindungsaffinitäten von $\beta$ - und $\gamma$-Globulinen sind für die untersuchten Corticoide sehr niedrig, aber für Fluocortolon und Difluocortolon höher als für Cortisol.
\end{abstract}

Diese Befunde könnten eine Erklärung für die hohe biologische Aktivität von $9 \alpha$-fluorierten Corticoiden, wie zum Beispiel Dexamethason, sein.

\section{Binding of cortisol, fluocortolone and difluocortolone to human plasma proteins}

Summary: The binding properties of $\left[{ }^{3} \mathrm{H}\right]$ cortisol, $\left[{ }^{3} \mathrm{H}\right]$ fluocortolone and $\left[{ }^{3} \mathrm{H}\right]$ difluocortolone by human plasma, human albumin, human- $\beta$ - and $\gamma$-globulins have been studied by equilibrium dialysis. Cortisol, in physiological concentrations $(0,4 \mu \mathrm{mol} / 1)$, is $98 \%$ bound in human plasma at $25^{\circ} \mathrm{C}$, fluocortolone $96 \%$ and difluocortolone $85 \%$. Under physiological conditions cortisol is mainly bound to the corticosteroid binding globulin (transcortin). $2 / 3$ of fluocortolone is bound to transcortin and $1 / 3$ to albumin and globulins, whereas difluocortolone is mainly bound to albumin and to globulins but not to transcortin. The binding affinities of $\beta$ - and $\gamma$-globulins are very low for the corticoids investigated, but they are higher for fluocortolone and difluocortolone than for cortisol.

These results could be an explanation for the high biological activity of $9 \alpha$-fluorocorticoids such as dexamethasone.

\section{Einleitung}

Die biologische Aktivität der Corticoide wird einerseits durch ihre Bindung an die Transportproteine, andererseits durch die an die zellulären Rezeptoren bestimmt. Zahlreiche Untersuchungen wurden bereits über die Bindung von Cortisol an Plasmaproteine (1), insbesonders an Albumin $(2,3,4)$, durchgeführt. Für den Transport im Blut (5) sind im wesentlichen Albumin (geringe Affinität bei hoher Kapazität) und Transcortin (hohe Affinität bei geringer Kapazität) verantwortlich. Um eine Information über die Struktur-Wirkungsbeżiehung fluorierter Corticoide zu erhalten, interessierten wir uns für den Einflußs der $6 \alpha$-bzw. $9 \alpha$-Fluorierung von Corticoiden auf deren Bindungsverhalten gegenüber den Transportproteinen. Für unsere Studie verwendeten wir $6 \alpha$-Fluocor- tolon und $6 \alpha, 9 \alpha$-Difluocortolon. Cortisol wurde als bekannte Referenżsubstanz mitbestimmt. Da entsprechend der Arbeiten von Brunkhorst \& Hess (6) die Albuminkonzentration die Gleichgewichtskonstante beeinflußt, arbeiteten wir mit einer den physiologischen Bedingungen in etwa entsprechenden Albuminlösung von $35 \mathrm{~g} / 1$. Die Corticoide wurden in physiologischen Konzentrationen $(0,1-1,5 \mu \mathrm{mol} / 1)$ und in stark erhöhten Konzentrationen (100-1500 $\mu \mathrm{mol} / \mathrm{l})$ eingesetzt.

\section{Material}

Cortisol (Serva 25 190) bzw. [1,2,6,7 - $\left.{ }^{3} \mathrm{H}\right]$ Cortisol (NEN), spezifische Aktivität: $3,37 \mathrm{TBq} / \mathrm{mmol}$ ( $91 \mathrm{Ci} / \mathrm{mmol}$ ), Reinheit: $98 \%$. 


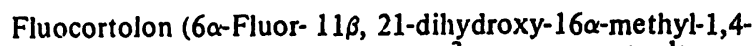
pregnadien-3,20-dion) bzw. $\left[1,2,4-{ }^{3} \mathrm{H}\right]$ Fluocortolon ${ }^{1}$ ) spezifische Aktivität: $18,5 \mathrm{GBq} / \mathrm{mmol}(0,4995 \mathrm{Ci} / \mathrm{mmol})$, Reinheit: $>98 \%$.

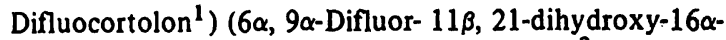
methyl-1,4-pregnadien-3,20-dion) bzw. $\left[1,2,4-{ }^{3} \mathrm{H}\right]$ Difluocortolon $\left.^{1}\right)$ spezifische Aktivität: $12,1 \mathrm{GBq} / \mathrm{mmol}(0,326 \mathrm{Ci} / \mathrm{mmol})$, Reinheit: $>98 \%$.

Die Proteinfraktionen wurden von Miles Laboratories bezogen: Human-Albumin krist. (Elektrophoretische Reinheit: 100\%); Human- $\beta-$ Globulin (Cohn Fr. 3), Human- $\gamma-$ Globulin (Cohn Fr. 2) rekrist.

Im verwendeten Plasma wurde mittels Cellulose-Acetat-Folienelektrophorese die Albuminkonzentration mit $32,2 \mathrm{~g} / 1$ und die Globulinkonzentration mit $23,8 \mathrm{~g} / 1$ bestimmt.

Dialyseschlauch: Visking 8/32 (regenerierte Cellulose). Phosphatpuffer $(0,1 \mathrm{~mol} / \mathrm{l} \mathrm{pH}=7,4)$. Szintillationsflüssigkeit: 51 Toluol, 2,5 1 Triton X-100 (Serva 37240), 41,2 g PPO (Merck 2946), $0,75 \mathrm{~g}$ Dimethyl-POPOP (Serva 15125).

\section{Methode}

Die von Brunkhorst \& Hess (6) beschriebene Methode der Gleichgewichtsdialyse wurde den uns zur Verfügung stehenden geringen Plasmamengen angepaßt. In Vorversuchen stellten wir fest, daß das Gleichgewicht bei Untersuchung der Proteinfraktionen nach längstens 8 Stunden und im Fall von Plasma nach längstens 18 Stunden eingestellt ist. Der Außenraum enthält den Phosphatpuffer mit dem ${ }^{3} \mathrm{H}$-markierten Corticoid. Im Dialyseschlauch befindet sich das zu untersuchende Protein. Die Menge gebundenen Corticoids wurde durch Messung der Radioaktivität im Innen- bzw. Außenraum ermittelt. Je $2 \times 0,4 \mathrm{ml}$ aus dem Innenbzw. Außenraum wurden gemessen. Quenchkorrekturen wurden mit der "Channel ratio"-Methode durchgefuhrt. Die Auswertung der Meßdaten erfolgte anhand der von Klotz angegebenen Gleichung (7).

$\frac{1}{r}=\frac{1}{n k} \frac{1}{C_{f}}+\frac{1}{n} \quad r=\frac{C_{g}}{P_{t}}$

$\mathrm{C}_{\mathrm{f}} \ldots$ Konzentration an freiem Corticoid

$\mathrm{C}_{\mathrm{g}} \ldots$ Konzentration an gebundenem Corticoid

$P_{t}$... Gesamtkonzentration an Protein

n ... Zahl der Bindringszentren/Proteinmolekül

k ... Affinitätskonstante

nk ... Maß für Affinität

Für den Fall, daß n gleiche, voneinander unabhängige Bindungszentren, vorliegen, muß eine Gerade resultieren, wenn

$\frac{1}{\mathrm{C}_{\mathrm{f}}}$ gegen $\frac{1}{\mathrm{r}}$ aufgetragen wird. Aus dem Schnittpunkt der Geraden mit der Ordinate erhält man $\frac{1}{n}$, aus dem Anstieg $\frac{1}{n k}$. Die Gerade, die den gemessenen Werten am ehesten entspricht, wurde mittels Regressionsanalyse bestimmt.

\section{Resultate}

\section{Humanalbumin/Corticoide}

Die Bindung der drei uns interessierenden Corticoide an Albumin wurde im physiologischen Bereich $(0,1-1,5$ $\mu \mathrm{mol} / \mathrm{l})$ und bei hohen Corticoidkonzentrationen (100-1500 $\mu \mathrm{mol} / \mathrm{l})$ untersucht. Nur beim Arbeiten mit hohen Corticoidkonzentrationen ist eine genaue Aussage

1) Wir danken der Firma Schering AG-Berlin für die freundliche Überlassung der Substanzen. über die Zahl der Bindungszentren (Tab. 1) möglich. Die Meßergebnisse im niedrigen Konzentrationsbereich sind in Abbildung 1 zusammengefaßt. Fluocortolon wird zu etwa $87 \%$, Difluocortolon zu etwa $77 \%$ und Cortisol zu etwa $58 \%$ an Albumin gebunden. Abbildung 2 zeigt die Bindungsverhältnisse bei 100 bis 1000 -fachen Corticoidkonzentrationen. Die nk-Werte (Tab. 1) liegen bei hohen Konzentrationen niedriger als bei physiologischen Corticoidkonzentrationen, aber nicht um einen konstanten Faktor.

\section{Globuline/Corticoide}

Um die Bindung dèr drei Corticoide an Humanglobuline zu studieren, wurden diese im Phosphatpuffer gelöst: $\beta$-Globuline $(8,5 \mathrm{~g} / 1) ; \gamma$-Globuline $(13,7 \mathrm{~g} / \mathrm{l})$; Die Untersuchungen wurden mit derselben Gleichgewichtsdialyseanordnung durchgeführt wie für Albumin. Im physiologischen Konzentrationsbereich ist weniger als $0,5 \%$ des Cortisols an die untersuchten Globuline gebunden; Fluo-

Tab. 1: n und nk-Werte für Cortisol, Fluocortolon und Difluocortolon. Die Konzentration an Humanalbumin beträgt $0,5 \mathrm{mmol} / \mathrm{l}$. Die Corticoidkonzentration für Spalte 1 beträgt 0,1 bis $1,5 \mu \mathrm{mol} / 1$; für Spalte 2 beträgt sie 45 bis $1000 \mu \mathrm{mol} / 1$; bei dieser Konzentration wurden auch die $\mathrm{n}$ Werte ermittelt. Die Berechnung erfolgte mittels Regressionsanalyse.

\begin{tabular}{lrll}
\hline & $\begin{array}{l}1 \\
n k( \pm 2 s) \\
{[1 / \mathrm{mmol}]}\end{array}$ & $\begin{array}{l}2 \\
\mathrm{nk}( \pm 2 \mathrm{~s}) \\
{[1 / \mathrm{mmol}]}\end{array}$ & $\mathrm{n}( \pm 2 \mathrm{~s})$ \\
\hline Cortisol & $2,86 \pm 0,44$ & $2,14 \pm 0,05$ & $3,13 \pm 0,28$ \\
Fluocortolon & $14,70 \pm 0,36$ & $7,88 \pm 0,30$ & $2,16 \pm 0,24$ \\
Difluocortolon & $6,52 \pm 0,15$ & $5,68 \pm 0,12$ & $2,85 \pm 0,36$ \\
\hline
\end{tabular}

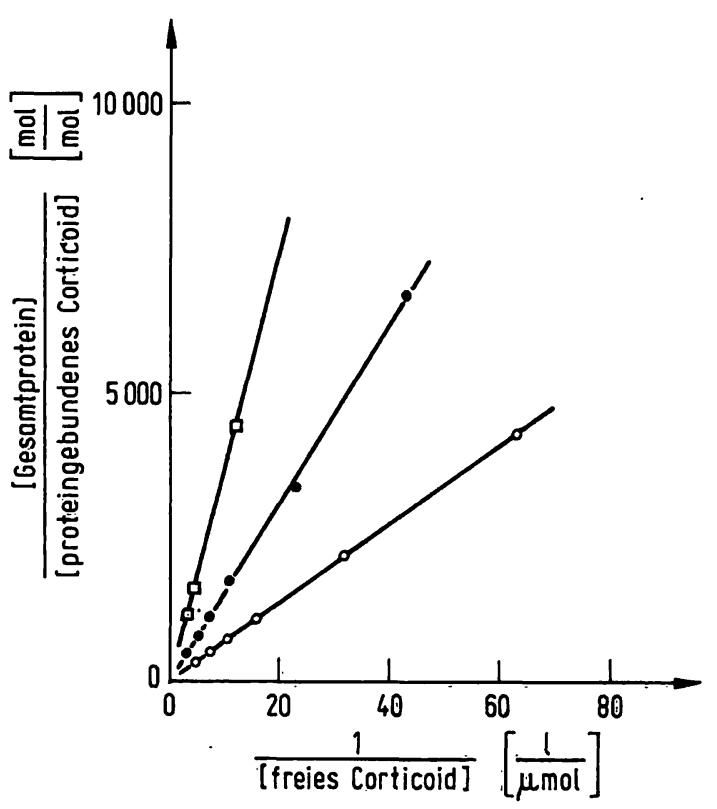

Abb. 1. Bindung von Cortisol (a), Fluocortolon. (o), und Difluocortolon $(\bullet)$ in physiologischer bzw. pharmakologischer Konzentration $(0,1-1,5 \mu \mathrm{mol} / \mathrm{l})$ an Serumalbumin vom Menschen, bestimmt mittels Gleichgewichtsdialyse bei $25^{\circ} \mathrm{C}$ in Phosphatpuffer. . . 


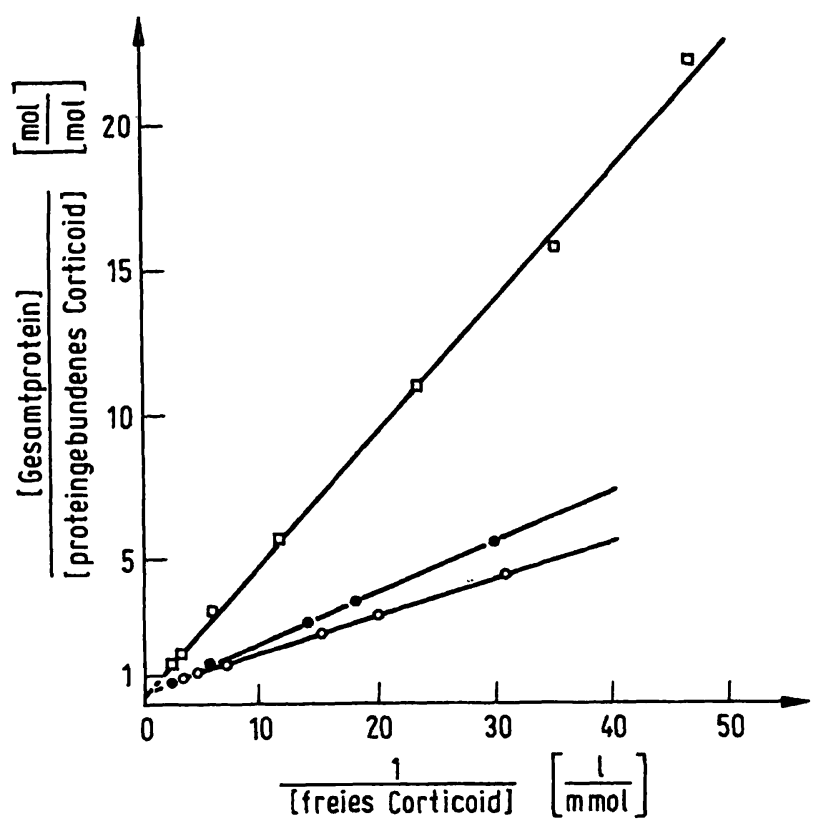

Abb. 2. Bindung von Cortisol (a), Fluocortolon (o) und Difluocortolon $(\bullet)$ in unphysiologisch hoher Konzentration (100-1500 $\mu \mathrm{mol} / \mathrm{l})$ an Serumalbumin vom Menschen, bestimmt mittels Gleichgewichtsdialyse bei $25^{\circ} \mathrm{C}$ im Phosphatpuffer.

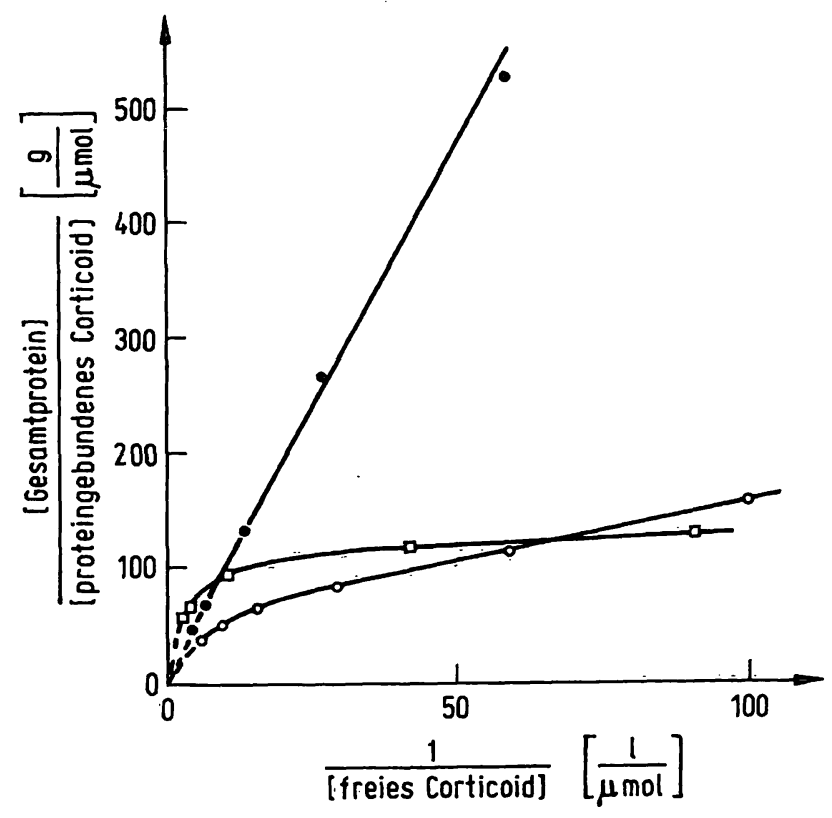

Abb. 3. Bindung von Cortisol (a), Fluocortolon (0) und Difluocortolon $(\bullet)$ in physiologischer Konzentration $(0,1-1,5$ $\mu \mathrm{mol} / \mathrm{l})$ an Humanplasma, bestimmt mittels Gleichgewichtsdialyse bei $25^{\circ} \mathrm{C}$ im Phosphatpuffer.

cortolon und Difluocortolon werden unter diesen Bedingungen bis zu $4 \%$ an Globuline gebunden.

\section{Humanplasma/Corticoide}

Es wurde Humanplasma untersucht, wie die Proteinfraktionen, allerdings nur bei physiologischen Konzentrationen der Steroide. Die Ergebnisse sind in Abbildung 3 dar: gestellt.
Die Gerade für Difluocortolon bedeutet, daß bei sämtlichen untersuchten Konzentrationen dieselben Bindungszentren maßgebend sind. Bei Cortisol zeigt sich bei höheren Konzentrationen ein steiler Kurvenverlauf, der der Bindung von Cortisol an Albumin entspricht. Bei Erreichen physiologischer Cortisolkonzentrationen wird Cortisol hauptsächlich an CBG gebunden, was den wesentlich flacheren Kurvenverlauf in diesem Bereich bedingt. Für Fluocortolon resultiert bei hohen Konzentrationen entsprechend der festeren Bindung an Albumin ein flacherer Kurvenverlauf als für Cortisol. Der noch flachere Kurvenverlauf bei physiologischen Konzentrationen resultiert aus der in diesem Bereich auch quantitativ bedeutenden Bindung an CBG.

\section{Diskussion}

Corticoide werden in Humanplasma an Proteine gebunden transportiert. Nur ungebundene Corticoide sind biologisch aktiv, beziehungsweise einer Metabolisierung und Ausscheidung zugänglich $(8,9,10)$; somit ist die Untersuchung der Corticoid-Protein-Wechselwirkung von Bedeutung. Wir untersuchten die Bindung von Fluocortolon und Difluocortolon an Humanplasmaproteine im Vergleich zu Cortisol, um zu einer Struktur-Wirkungsbeziehung zu kommen. Die beiden untersuchten Steroide unterscheiden sich voneinander lediglich durch den $9 \alpha$ Fluorsubstituenten. Gerade der Einfluß dieses Substituenten ist recht interessant, studiert zu werden, zeichnen sich doch Corticoide mit $9 \alpha$-Fluorsubstitutent (Dexamethason, Betamethason, Triamcinolon) durch besonders hohe biologische Aktivität aus. Durch unsere Untersuchungen wurde eine Bindung von Fluocortolon an Transcortin nachgewiesen, während eine solche für Difluocortolon nicht beweisbar ist. Somit scheint durch die Einführung des $9 \alpha$-Fluoratoms die Bindung an Transcortin inhibiert zu werden, wie ja auch eine Bindung von Dexamethason an Transcortin nicht meßbar ist. Dieser Befund wäre zusammen mit der Feststellung, daß $9 \alpha$ fluorierte Corticoide stärker an die cytoplasmatischen Receptoren gebunden werden als Cortisol $(11,12)$, eine Erklärung für deren stark erhöhte biologische Wirksamkeit.

Wie Brunkhorst \& Hess (4) für Cortisol, konnten wir für die von uns untersuchten Corticoide zeigen, daß bei niedrigen Corticoidkonzentrationen eine stärkere Bindung an Albumin vorliegt als bei höheren. Möglicherweise sind Konformationsänderungen, ähnlich den von Stroupe \& Westphal (13) für das Progesteron-bindende Globulin beschriebenen, dafür verantwortlich. Jedenfalls erklärt dieser Konzentrationseinfluß die unterschiedlichen Angaben, die in der Literatur $(3,4,5)$ für die Affinitätskonstante für das System Cortisol-Albumin gefunden werden. Darüber hinaus allerdings bringt die Abhängigkeit der nk-Werte von der Corticoidkonzentration eine Unsicherheit bei der Berechnung der Zahl der Bindungszentren mit sich, da eine solche lediglich aus Messungen bei 
hoher Konzentration durchgeführt werden kann. Sicher ist allerdings, daß bei niedriger Konzentration nicht mehr Zentren als bei hoher besetzt werden. Die Untersuchungen an Humanplasma zeigen, daß Difluocortolon fast ausschließlich an Albumin gebunden wird, während Cortisol und Fluocortolon bei physiologischen Konzentrationen vorwiegend an Transcortin angelagert werden und erst nach Absättigung des Transcortins von Albumin gebunden werden. Aus dem Kurvenverlauf ist auch die stärkere Bindung von Fluocortolon an Albumin im Vergleich zum Cortisol ersichtlich. Bei der Beurteilung der physiologischen Aktivität der untersuchten Corticoide aufgrund unserer Meßdaten muß man allerdings beachten, daß unsere Untersuchungen in einem statischen System in vitro durchgeführt wurden, daß für die physiologische Wirkung aber kinetisch kontrollierte Vorgänge bei Gleichgewichtsreaktionen im Organismus von wesentlicher Bedeutung sind (14).

\section{Danksagung}

Die Arbeit wurde mit Unterstïtzung des „Fonds zur Förderung der wissenschaftlichen Forschung "Wien, durchgeführt.

\section{Literatur}

1. Westphal, U. (1971), Steroid Protein Interactions, S. 107-114, Springer-Verlag Berlin-Heidelberg-New York.

2. Daughaday, W. H. (1958), J. Clin. Invest. 37, 519-523.

3. Scholtan, W., Schlossmann, K. \& Rosenkranz, H. (1968), Arzneim.-Forsch. 18, 767-780.

4. Brunkhorst, W. K. \& Hess, E. L. (1965), Arch. Biochem. Biophys. 111, 54-60.

5. Kripalani, K. J. \& Sorby, D. L. (1967), J. Pharm. Sci 56. 687-693.

6. Brunkhorst, W. K. \& Hess, E. L. (1964), Biochim. Biophỳs. Acta 82, 385-393.

7. Klotz, I. M. (1946), Arch. Biochem. Biophys. 9, 109-117.
8. Slaunwhite Jr., W. R., Lockie, G. N., Back, N. \& Sandberg, A. A. (1962), Science 135, 1062 $=1063$.

9. Sandberg, A. A. \& Slaunwhite Jr., W. R. (1963), J. Clin. Invest. 42, 51-54.

10. Anderson, J. N., Peck, E. J. \& Clark, H. J. (1974), J. Steroid Biochem. 5, 103-107.

11. Gardner, D. G. \& Wittliff, J. L. (1973), Biochim. Biophys. Acta 320,617-627.

12. Goral, J. E. \& Wittliff, J. L. (1975), Biochemistry 14, 2944-2952.

13. Stroupe, S. D. \& Westphal, U. (1975), Biochemistry 14, 3296-3300.
Priv. Doż. Dr. H. Gleispach Univ.-Kinderklinik A-8010 Graz 